gläserner Meßgefäße meist bei $18,17,5$ oder $15^{\circ}$ bestimmt. Die Polarisationsdrehung wässeriger Lösungen wird vorwiegend bei $20^{\circ}$, die Viskosität solcher meist bei $25^{\circ}$, chemische Gleichgewichte und Reaktionsgeschwindigkeiten werden bei 15, 18, 20, $25^{\circ}$ und anderen Temperaturen gemessen. Verhältnismäßig große Ubereinstimmung herrscht bei der Bestimmung des elektrischen Leitvermögens wässeriger Lösungen, fiur das im Gebiet der Zimmertemperatur nach dem Vorgange von Kohlrausch $18^{\circ}$ oder nach dem von Ostwald $25^{\circ}$ als Normaltemperatur bemutzt wird. Von den galvanischen Normalelementen ist bekanntlich das Clarkelement auf $15^{\circ}$, das Cadmiumelement auf $20^{\circ}$ bezogen.

Es ist klar, daß diese Verhältnisse Unzuträglichkeiten mit sich bringen. Die für eine Temperatur geeichten Maßgefäße oder Geräte können bei genauen Messungen nicht ohne weiteres für andere Temperaturen benutzt werden. Für Anbringung von Korrekturen wegen der Temperaturverschiedenheit fehlen häufig genaue Unterlagen. Oft wird der Beobachter veranlabt, seine Untersuchungen bei einer anderen als der gewünschten Temperatur zu machen, nur weil er sich nach der Temperatur richten muB, für die gewisse Eigenschaften der benutzten Stoffe schon früher gemessen worden sind.

Auch für die Technik besteht das dringende Beduirfnis nach Vereinbarungen über eine Normaltemperatur, wofür in den Exläuterungen mehrere Beispiele gegeben sind.

Bei der Wahl einer einheitlichen Zimmertemperatur könnte man für die Gebiete der reinen Physik und Chemie zwischen den bisher am meisten angewandten Temperaturen 18 und $20^{\circ}$ schwanken. Für $18^{\circ}$ liegt ein ungeheures Zahlenmaterial an physikochemischen Messungen der verschiedensten Stoffe vor. Indessen spricht gegen $18^{\circ}$ der Umstand, daB diese Temperatur in Deutschland im Sornmer meist nicht ohne künstliche Kühlung aufrecht zu erhalten ist; noch mehr gilt dies für die südlicher gelegenen Arbeitsstätten, die sich in immer steigender Zahl an genauen Messungen beteiligen. Da zudem seitens der Elektrotechniker eine internationale Vereinbarung auf der Grundlage von $20^{\circ}$ abgeschlossen ist, so empfiehlt es sich, dieser Wahl zu folgen.

Es versteht sich von selbst, daß der Physiker und Chemiker auch weiterhin bei wissenschaft lichen Forschungsarbeiten sich in den seltensten Fällen mit Messungen bei einer einzigen Temperatur begnügen wird, da er auch den Temperaturverlauf der betreffenden Werte zu ermitteln streben wird. Doch erscheint es entbehrlich, hierfür bestimmte Vorschläge zu machen. Es genügt, wenn die Messungen dieser Art jedenfalls unter anderem auch bei $20^{\circ}$ vorgenommen werden und wenn diese letztere Temperatur bei praktischen Messungen, z. B. bei technischen
Prüfungen, bei Analysen usw. allgemein angewendet wird.

Es versteht sich weiter von selbst, daß Fälle denkbar sind, in denen besondere Gründe für die Wahl anderer Temperaturen sprechen. Solche Fälle, in denen man sogar notwendigerweise die Bezugstemperaturen $0^{\circ}$ und $4^{\circ}$ beibehalten muB, sind oben im Entwurf aufgeführt.

Entwurf XVIII: Feld und Flup. 1. Den Raum, in welchem sich elektrische und magnetische Erscheinungen abspielen, bezeichnet man allgemein als elektromagnetisches Feld. Beschränkt sich die Betrachtung im besonderen auf die elektrischen oder auf die magnetischen Erscheinungen, so spricht man von einem elektrischen oder magnetischen Felde.

2. Das Integral der Normalkomponente eines Feldvektors über eine Fläche bezeichnet man als Fluß des Vektors durch die Fläche.

Im besonderen bezeichnet man das Integral der Normalkomponente der magnetischen Induktion über eine Fläche als Induktionsfluß und das Integral der Normalkomponente der dielektrischen Verschiebung über eine Fläche als Verschiebungsflub.

3. Den Induktionsflub durch eine von allen Windungen einer Spule umrandete Fläche bezeichnet man als Spulenfluß. Der FluB durch die Fläche einer einzelnen Windung heißnt Windungsflus.

Über die Erläuterungen zu den Sätzen des Entwurfs möge in einex der Originalveröffentlichungen nachgelesen werden. In den Erläuterungen wird u. a. hervorgehoben, $\operatorname{da} \beta$ der Fluß ein Skalar ist. Hieraus ergibt sich, daß der FluB durch eine bestimmte Fläche stets zahlenmäßig angegeben werden kann, während das Feld eines Vektors nur das Wirkungsgebiet bezeichnet, in dem der Vektor vorherrscht.

\section{Die Regeneration der Gonophore bei den Hydroiden ${ }^{1}$ ).}

\author{
Von Dr. Herbert Constantin Müller, Königs- \\ berg i. Pr.
}

Es ist von jeher aufgefallen, daB die Fähigkeit der Regeneration nicht allen Geschöpfen in gleich starkem Maße zukommt, und daß sie im großen und ganzen bei den niedersten Tiergruppen am stärksten auftritt und mit der steigenden Organisationshöhe der einzelnen Tierstämme an Kraft abnimmt. So vermögen die einzelligen Tiere stets die ihnen abgeschnittenen Teile wieder nou zu bilden, wenn in dem übrigbleibenden Teile ihres Leibes ein Stïck des Zellkernes vorhanden ist; die Schwämme und Nesseltiere, die zum Teil eine recht beträchtliche Größe er-

1) Den ausführlichen Bericht über dieses Thema siehe „Archiv für Entwicklungsmechanik der Orga nismen" Bd. 37 u. 38 . 
reichen, können aus sehr kleinen Stücken ihres Körpers (bis zu $1 / 7 \mathrm{~mm}$ Durchmesser) wieder den vollständigen Organismus aufbauen; die Würmer vermögen noch nach Halbierung sich wieder zu mehr oder weniger vollständigen, immerhin aber lebensfähigen Exemplaren zu ergänzen.

Eine solche Fähigkeit fehlt den Gliedertieren schon vollständig, obgleich unter ihnen noch einige (Pantopoden) auch nach so schweren Verletzungen wie Querteilung am Leben bleiben und zur Regeneration wenigstens den Ansatz machen; sie vermögen in den meisten Fällen wenigstens noch einzelne Gliedmaßen und dgl. zu regenerieren. Den höchst entwickelten Tieren aber geht auch diese Eigenschaft immer mehr verloren, und wir können es an unserem eigenen Leibe verspüren, daß wir nicht imstande sind, auch nur relativ geringe Gewebeverluste zu ersetzen; beim Menschen beschränkt sich die Regenerationsfähigkeit auf die Körperhaut und ihre Gebilde und auf kleinere Gewebsneubildungen, wie sie zum Beispiel zur Schließung von Wunden erforderlich sind.

Es ist also festgestellt worden, daß das Regenerationsvermögen sich der Differenziertheit der einzelnen Tierstämme anpaßt; aber man kann diese Beziehungen noch innerhalb der einzelnen Tiergruppen verfolgen. Nahverwandte Organismen sind stets durch ein ungefähr gleiches Régenerationsvermögen ausgezeichnet, doch sind die einfachsten Tiere jeder Gruppe stets regenerationsfähiger als ihre differenziertesten Spitzen. Bei den Nesseltieren (Coelenteraten) z. B. haben die Hydroidpolypen ein ganz auBerordentliches Regenerationsvermögen; die zu demselben Stamm gehörigen Quallen aller Arten besitzen dieses Vermögen sehr viel weniger oder gar nicht. Wie einfach die Hydroidpolypen und wie kompliziert die Quallen gebaut sind, will ich später noch genauer auseinandersetzen. Die Bedeutung der Tatsache, daB die Regenerationskraft mit der fortschreitenden Differenzierung der Organismen immer mehr abnimmt, liegt darin, daß man aus ihr geschlossen hat, die Regenerationsfähigkeit sei eine ursprüngliche Eigenschaft der lebenden Substanz, die erst mit der Entwicklung der einfachen Lebewesen zu differenzierteren an Stärke immer mehr abgenommen hat, und nicht, wie man wohl von vornherein vermutete, allmählich durch Selektion erworben worden ist. DaB auch Ausnahmen der besprochenen Erscheinung bemerkt worden sind, fällt wenig ins Gewicht; denn von diesen Ausnahmen haben sich bei genauer Prüfung so viele als irrtümlich erwiesen, daß man annehmen kann, sie werden alle fortfallen.

Wine andere sehr weit verbreitete regelmäBige Erscheinung ist die, daB im Alter beim ausgewachsenen Tier die Fähigkeit zur Regeneration geringer wird, als sie das junge Tier zeigt, und nicht nur das allgemeine Regenerationsvermögen, sondern auch die Geschwindigkeit des
Prozesses nimmt im Alter ab. Ganz besonders auffällig wird dies an Tieren, die ihre Entwicklung über ein Larvenstadium vollziehen, wobei die Larven oft das erstaunlichste Regenerationsvermögen zeigen, die ausgewachsenen Tiere aber nur noch ein sehr schwaches.

Man muB annehmen, daB die Regeneration den Tieren nützlich sei. Man bedenke, welche Folgen das Ausbleiben der sogenannten physiologischen Regeneration, also der Ersatz der Haare, Nägel, Klauen, Hautteile und dgl. haben würde; welche Schädigungen viele niedere Tiere durch das freiwillige Abwerfen von Körperteilen in Gefahren erleiden würden, wenn sie dieselben nicht wieder ersetzen könnten; wie stark das Leben und die Arterhaltung vieler einzelliger Tiere und der festsitzenden Schwämme und Coelenteraten u. a. m. bei der Unzahl der Feinde bedroht wären, wenn ihnen allen nicht die Fähigkeit gegeben wäre, anch aus kleinen Uberresten wieder ganze Tiere aufzubauen.

Die oben erwähnten und noch manche andere Erfahrungen, deren Bedeutung für die Erforschung des Regenerationsgeschehens nicht zu verkennen noch zu unterschätzen ist, sind nur aus Resultaten gewonnen, die im Hinblick auf die unübersehbare Formenfülle des Tierreiches verschwindend gering sind, und sie lassen sich eben wegen ihres Charakters als oft wiederkehrende Erfahrung nur in Regeln formulieren, nicht aber in ausnahmslosen Gesetzen. Da sich bisher die meisten Ausnahmen dieser Regeln, namentlich der erst erwähnten, daß die Güte des Regenerationsvermögens sich nach der Höhe der Organisation und Differenzierung richtet, haben widerlegen lassen, so besteht die Möglichkeit, diese Regel noch immer ausnahmslos zu machen und sie nach tieferem Eindringen in ihr Verständnis zu einem Gesetze zu erheben.

$\mathrm{Es}_{\mathrm{s}}$ ist also die Aufgabe gestellt, diese Regel in möglichst vielen Fallen genau zu prüfen und auf nähere Details dabei einzugehen, um zu erfahren, wie zuverlässig das Regenerationsvermögen. von der Organisationshöhe bestimmt wird. Hierfür gibt es in der ganzen Tierwelt kein geeigneteres Studienobjekt als die schon oben erwähnten Hydromedusen.

Von diesen besitzen die Hydroidpolypen eine große Regenerationskraft. Sie sitzen - zumeist im Meere - an Steinen oder anderen Gegenständen des Untergrundes fest und bilden kleine, baumartig verzweigte Kolonien mit oft sehr vielen Einzelindividuen daran, den sogenannten Hydranten. Das Gewebe des Hydranten sowohl wie des sie tragenden verzweigten oder wnverzweigten Stammes ist sehr einfach zusammengesetzt. Es besteht aus nur zwei Körperschichten, die voneinander weder in der Stärke, noch im Bau oder Aussehen beträchtlich abweichen (vgl. Fig. 1). Auch ihre Funktionen sind verhältnismäßig wenig spezialisiert. Nach auBen hin werden diese beiden Körperschichten von einer 
durchsichtigen, zähen Hülle umgeben. Das Einzeltier, der Hydrant, unterscheidet sich von dem glatten Gewebe des Stammes nur sehr wenig; er ist schlicht sackförmig und hat am frei endenden Pol eine kleine Offnung, den Mund, um den herum lange, feine Fangarme, wiederum aus beiden Körperschichten gebildet, stehen (Fig. 1). Alles in allem sind also Stamm und Hydrant von einem sehr primitiven, einfachen Bau, fast ohne jede Komplizierung. Das Regenerationsvermögen beider ist bedeutend. Aus einem Fangarme, an dem nur ein kaum sichtbarer Rest des Hydrantenleibes hängt, kann ein neues Tier entstehen. Bei Hydra (Fig. 1), einem einzeln lebenden SüBwasser-

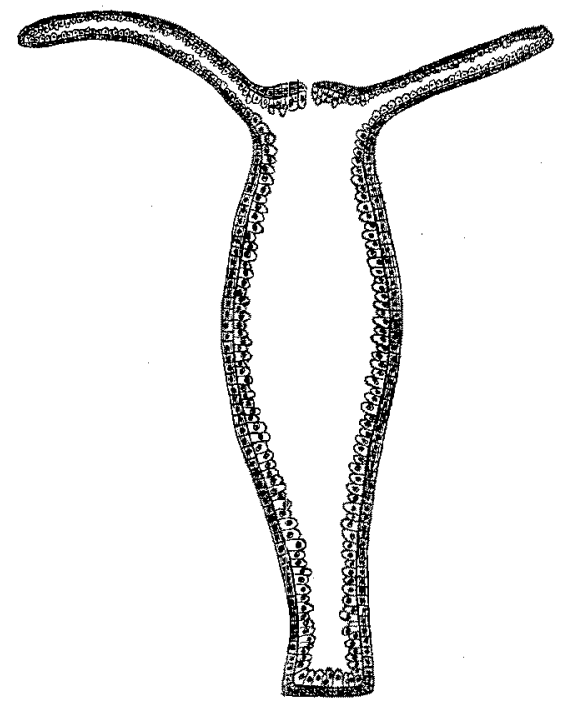

Fig. 1. Längsschnitt durch Hydra, einen solitär lebenden Hydroidpolypen als Typus eines Hydranten.

polypen, ist zur Regeneration des ganzen Tieres (ca. $1 \mathrm{~cm}$ Größe) nur eine Gewebekugel von etwa $1 / 6 \mathrm{~mm}$ Durchmesser erforderlich. Natürlich ist das Regenerationsvermögen nicht bei allen Hydroidpolypen gleichmäßig stark ausgebildet, im großen und ganzen sind aber die eben angeführten Beispiele ziemlich typisch dafür, und allzu starke Abweichungen kommen nicht vor.

Anders verhält es sich dagegen mit den Quallen, die von diesen Hydroidpolypen aufgeammt verden, d. h. an der Körperwand als kleine Knospen entstehen, sich auswachsen und dann nach Loslösung rom Muttertier ein selbständiges Leben führen. Diese Quallen besitzen gar kein oder ein sehr schwaches Regenerationsvermögen. (Nur eine ältere Arbeit Häckels berichtet über ein ziemlich weitgehendes Regenerationsvermögen bei den Thaumantiaden, einer Gruppe dieser Medusen; alle übrigen Versuche haben ergeben, daß wohl eine Regulation, d. h. Zusammenziehung, Wundheilung und wohl auch eine Umformung des operierten Tieres zur Herstellung der alten Körperform, aber keine Regeneration stattfindet. Im übrigen sind die Berichte über dieses spezielle Thema nur sehr spärlich vorhanden, was wohl seinen Grund in den negativen Resultaten der operativen Versuche hat, da man sich bei der Jugend der Regenerationsforschung naturgemäß zunächst auf die lohnendsten und ergiebigsten Untersuchungen beschränken mußte.) Mit dem schwächeren Regenerationsvermögen der Medusen gegenüber den Hydroidpolypen stimmt es überein, daB jene ungleich komplizierter und differenzierter gebaut sind als diese. Jene zarten Gebilde, die im Wasser umherflottieren, bestehen zwar im Prinzip auch nur aus zwei Körperschichten, diese sind jedoch ungleich mehr differenziert und spezialisiert (vgl. auch Fig. 2), und zwischen beide schiebt sich in der Glocke, dem Hauptteil des Körpers, noch ein gallertiges, meist an Umfang sehr beträchtliches Zwischengewebe ein. Die Medusen haben ein wohl ausgebildetes Nervensystem, Sinnesorgane, Schwimmeinrichtungen usw, alles Dinge, die den Hydroidpolypen vollständig abgehen. Die Medusen sind in erster Linie dazu bestimmt, Geschlechtsprodukte zu erzeugen und diese, weil sie frei beweglich sind, an Orten, die rom Mutterpolypen entfernt sind, abzugeben.

Nun gibt es aber zahlreiche Fälle, in denen die Medusen infolge der zu raschen Reifung ihrer Geschlechtsprodukte nicht mehr dazu kom. men, sich vom Stamm der Amme loszulösen. Weiterhin ist es die Mehrzahl aller Formen, bei denen zwar medusenartige Knospen vom Hydroidpolypen angelegt werden; aber nicht mehr zur völligen Ausbildung einer Meduse gelangen, sondern nur zu einem mehr oder weniger primitiven Stadium, das aber in den meisten Fällen noch irgendwelche Verwandtschaft mit der frei schwimmenden Meduse zeigt. Dies sind die sogenannten Gonophore; unter ihnen findet man alle Ưbergänge von der fast völlig ausgebildeten Meduse bis zum einfachen Schlauch, in dessen Wandungen die Keimmassen eingebettet liegen (vgl. auch Fig. 3). Alle diese Gonophore haben außer der von Stufe zu Stufe verfolgbaren Verwandtschaft mit den Medusen auch noch mit diesen eine besonders typische, komplizierte Entwicklung über ein sogenanntes Glockenkernstadium gemein. Nur wenige solcher Gonophore gibt es, die sowohl im Bau als auch in der Entwicklung von der Allgemeinheit abweichen; zu diesen gehören die nachher zu besprechenden Geschlechtsorgane der Cordylophora lacustris.

Wir haben also zwei Extreme, den einfachen Polypen und die komplizierte Meduse, von denen das eine sehr gut regeneriert, das andere sehr schlecht, und dazwischen sehr viele Übergänge, die von der komplizierten Meduse nach einem immer einfacheren Zustande hinzielen, der dem des Polypen nicht mehr allzu fern ist. An diesen Übergängen ist nun ein ungemein reichhaltiges Material gegeben, das Zusammenlaufen der Regenerationskraft mit der Niedrigkeit der Organisation Schritt für Schritt zu verfolgen. Gleichzeitig kann man den EinfluB des. Alters auf die Regene- 
ration untersuchen, man ist in den einzelnen Operationen sogar dazu gezwungen, weil die sessilen Gonophore nie lange im erwachsenen Zustande verharren, sondern die während des eigenen Wachstums gereiften Geschlechtsprodukte möglichst bald entleeren und dann wieder vom Stamme aufgesogen werden. Operative Eingriffe muB man also tunlichst an wachsenden Stadien vornehmen. Dabei ist zu beachten, daß sessile Gonophore und Medusenknospen wohl in ibren letzten Entwicklungsstadien mehr oder weniger voneinander verschieden sind, dab sie sich aber alle in den Anfängen ihrer Entwicklung, eben in dem oben erwähnten Glockenkernstadium, sehr ähnlich sind. Auch insofern konnte die Bearbeitung der Gonophore und Medusenknospen sehr viel Interessantes bieten, als die Bedeutung dieser Organe eine sehr große ist, da die ganze geschlechtliche Fortpflanzung auf ihnen beruht.

Bei der Regeneration der kleinen, an den $\mathbf{H y}$ droidpolypen sitzenden Medusenknospen und Gonophoren muBte man prinzipiell zweierlei unterm scheiden: einmal ob die ganzen Gebilde vom Stamm regeneriert werden, wozu sie also dicht an ihrer Ansatzstelle abgeschnitten werden müssen, und andrerseits ob die Gebilde selbst imstande sind, verlorengegangene eigene Gewebeteile zu ersetzen. Denn es ist klar, daß im ersten Falle der Hydroidpolyp, im zweiten aber das Gonophor oder die Medusenknospe selber die Regeneration bewerkstelligen müssen. Der praktische

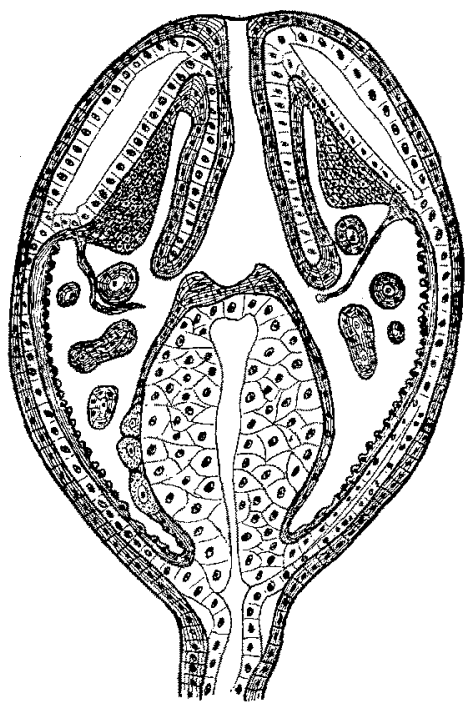

Fig. 2. Längsschnitt durch eine ausgewachsene Medusenknospe von Podocoryne carnea (nach Goette).

Erfolg zeigte, daß beides stets Hand in Hand geht; wo die ganzen Gonophore nicht vom Stamme regeneriert werden, können sie auch selber verlorene Teile nicht wieder neu bilden, und wo das ganze Gebilde vom Stamme aus regeneriert wird, kann es auch selber verlorene Teile wiedex neu bilden, - Wie es zu erwarten war, regenerieren die Medusenknospen (Fig. 2), also die wach- senden Gebilde, aus denen später die frei lebenden Quallen werden, nicht. Schnittwunden an ihnen heilen zwar zu, wozu ja auch (in sehr beschränktem $\mathrm{MaBe}$ ) Gewebeneubildungen erforderlich sind, und die verstümmelten Objekte stellen durch Zusammenziehen und Unformung wieder eine geschlossene Form her - alles, wie es auch bei freilebenden Medusen geschieht, aber nicht mehr. Aber nicht nur den Medusenknospen, sondern auch den Gonophoren aller Organisationsstufen mit Glockenkernentwicklung, die ich untersuchte, fehlt das Vermögen einer Regeneration. Wie es die Fig. 3

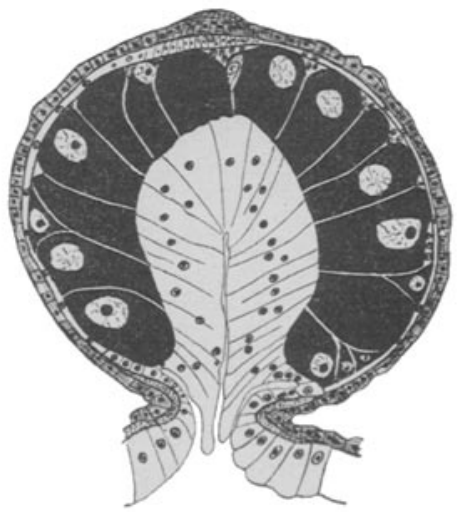

Fig. 3. Längsschnitt durch ein Gonophor von Coryne frutieosa (nach $K$ ïhn).

zeigt, bildet das Keimlager in diesen Gonophoren meistens die Hauptmasse des Gewebes; und dieses Keimlager ist es in erster Linie, das nicht regeneriert. Die übrigen somatischen Teile der Gonophore sind dagegen mehr oder weniger regenerationsfähig; trotzdem verhindert das Keimlager durch seine Passivität jede gröliere Regeneration. Die regenerative Fähigkeit der übrigen Gewebeteile, in Verbindung mit ihrer meist ungemein starken Umbildungsfähigkeit, ermöglicht es aber, daß nach schweren Eingriffen in das Gonophor dieses sich mehr oder weniger gut reguliert, d. h. seine alte Form wiederherstellt; so kann beispielsweise ein eiförmiges Gonophor, das in der Mitte quer durchschnitten wurde, durch die Tätigkeit der somatischen Teile bald wieder eine eiförmige Gestalt annehmen, so dab wir schließlich ein Gonophor der alten, normalen Form, aber nur in der halben Größe erhalten. Dabei können beschränkte Gewebepartien, z. B. Teile der äuBeren Umhüllung, Befestigungsbänder und dgl., mitunter auch Stücke der schützenden Chitinhülle neu gebildet werden. Nicht überall ist die eigene Regenerationsfähigkeit der somatischen Teile und ihre Umwandlungsfähigkeit gleich stark ausgebildet; im groben und ganzen aber weichen die Gonophore darin nicht allzu stark voneinander ab. -

Ich muB hier auch noch gleich der ,Blastostyle" der Thecaten (eine der beiden Hauptgruppen der Hydroiden) gedenken. Bei diesen sitzen in den meisten Fällen die Gonophore und 
Medusenknospen in dichten Massen an besonderen Gebilden, den Blastostylen, die ihrem Aufbar nach einige Ahnlichkeit mit den Hydranten haben; sie sind zweischichtig, "wobei diese Körperschichten sehr dünn sind und keine direkten Funktionen mehr ausüben. An thnen sitzen dicht gedrängt die geschlechtlichen Gebilde. Auch diese Blastostyle sind wie die Gonophore nicht imstande zu regenerieren, d. h. z. B. nach Querschnitten ihre alte GröBe wiederherzustellen. Wahrscheinlich werden sie hieran durch die enormae Zahl der Geschlechtsgebilde, die sie beständig hervorbringen, verhindert. Ziemlich beträchtliche Gewebe- und Chitinneubildungen konnten aber an thnen konstatiert werden. Wo bei den zahlreichen Versuchen an Blastostylen sehr vereinzelt doch einmal Regeneration auftrat - es geschah dies nur an besonders kräftigen Kolonien -; da regenerierte nicht "das ursprüngliche Gebilde, sondern stets ein solches, das die typische Struktur des regenerationsfähigen Stammes besaB und als Wurzelfaden angesprochen werden muß. Von dem gemeinschaftlichen regenerativen Verhalten der Gonophore und Medusenknospen weicht ein einziges in allen den vielen untersuchten Fillen prinzipiell ab, das ist das weibliche Gonophor der Cordylophora lacustris. Es regeneriert. verlorene Stücke, anch sehr grobe, rasch und sicher, und ebenso wird das ganz abgeschnittene Gonophor rom Stamm sofort neu gebildet. Den männlichen Gonophoren der Cordylophora seheint die Regenerationsfähigkeit ebenso zu fehlen, wie den Gonophoren und Medusenknospen aller übrigen Formen; jedoch sind die Tntersuchungen über diesen Punkt noch nicht mit vollkommener Sicherheit beendet, so daß sich möglicherweise diese Tatsache als irrig erweisen kann.
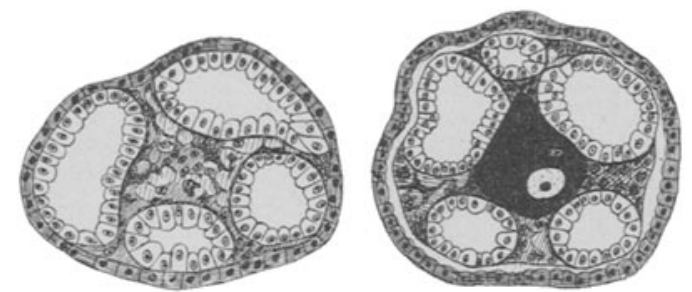

Fig. 4. Zwei Quersehnitte in versehiedenen Höben durch ein weibliches Gonophor von Cordylophora laoustris. (nach Goette).

Ein Vergleich zwischen den Gonophoren de: Cordylophora und denen der anderen untersuchten Hydroiden zeigt uns nun wesentliche, prinzipielle Unterschiede zwischen beiden. Während die Gonophore im Prinzip gewöhnlich so gebaut sind (Fig. 3), daß ein Zapfen der inneren Körperschicht vou einem dicken Keimlager, welches seinerseits von äuBerst dünnen Hüllen der äuBeren Körperschicht umschlossen wird, sehen wir bei Cordylophora (Fig. 4) diese äuBere Hülle sehr viel stärker, kräftiger auftreten, der Zapfen der inneren $K$ örperschicht ist in viele große Aste geteilt, die zum Teil direkt an die äuBere Umbülung stoßen; außerdem liegen die Geschlechtszellen nieht direkt zwischen den beiden Körperschichten eingebettet, sondern sie sind in einem somatischen Zwischengewebe ziemlich weit verteilt und deshalb auch verhaltnismäBig nicht so mächtig und überwältigend ausgebildet, wie bei den übrigen Gonophoren. Dazu kommt noch, dals die Cordylophora-Gonophore eine direkte, einfache Entwicklung, ähnlich wie der Hydrant, durchmachen und das typische, komplizierte Glockenkernstadium vermeiden. Dieses so beschaffene Gonophor der Cordylophora lacustris nun regeneriert! Damit wäre festgestellt, daß sich die Regeneration in gewissem Maße tatsächlich an die feineren Unterschiede der Struktur bei den Gonophoren der Hydroiden hält. - Trotzdem die Cordylophora-Gonophore uns aber dies Resultat an die Hand geben, sind die Erwartungen, die man hätte hegen dürfen, nicht erfüllt worden. Man hätte sich wohl nicht gewundert, wenn sich die Regeneration den einzelnen Štufen der Gonophorhöhe angepaßt und sich so den Einzelheiten der Differenzierung innig angeschmiegt hätte. Statt dessen zeigt sie sich nur ganz grob daran gebunden, und ob wir in diesem einzelnen Falle nicht auch noch einen Zufall zu sehen haben, ist nicht einmal so sicher, da es eben nur ein einzelner Fall erfolgter Regeneration ist. - Übrigens sind auch die Abstufungen einer gewissen individuell-selbständigen Kraft, die sich während des Experimentierens an den Gonophoren und Medusenknospen beobachten ließ, durchaus nicht mit dem Orsanisationszustand der Gonophore verknüpft.

Weiterhin wird der Leser schon vermutet haben, daß auch das Alter für die Regeneration in unserem Falle durchaus keine Rolle spielt. Es war auch nie der leiseste Unterschied zu bemerken, ob ein junges oder ein ausgewachsenes $\mathrm{Ob}$ jekt durchschnitten wurde, bei Cordylophora sowohl wie bei den anderen Formen. Es sei hierbei erwähnt, daß, abgesehen von Cordylophora, sich die jungen Stadien der Gonophore und Medusenknospen meist zum Verwechseln ähnlich sehen. Die Regel also, daß die Regeneration mit dem Alter abnehme, oder mit anderen Worten gesagt, daß sie in der Jugend gröBer sei als im Alter, kommt in unserem Falle nicht zur Geltung.

Aus dem Nichtregenerieren der Gonophore bei fast allen Hydroiden muß man auch schließen, daB hier die Regenerationskraft nicht in einer für die Erhaltung der Art nützlichen Weise ausgebildet ist. Denn dazu müßsten die Medusenknospen und sessilen Gonophore in erster Linie regenerationsfähig sein, da bei ihnen ja ausschließlich die ungemein wichtige geschlechtliche Vermehrung liegt. Es muB andrerseits dabei auch wieder eingesehen werden, daß in der Natur ein Bedürfnis für dio Regeneration dieser Teile durchaus nicht vorliegt. Rein mechanische Störungen werden nie die kleinen zum Teil geschützt liegenden Gonophore 
allein treffen, sondern stets größere Teile des Stammes oder Blastostyles; dann aber werden mit diesen regenerierenden Stockteilen auch die Geschlechtsorgane mit neu gebildet, wofür experimentelle Beweise schon vorhanden sind. Feindliche Tiere aber werden sich, zumal bei den Athekaten, stets mehr an die ungeschützten Teile der Kolonie halten und die durch starkes und zähes Chitin geschützten Geschlechtsknospen umgehen. Wenigstens habe ich nie das Fehlen eines einzelnen Gonophors in der Natur bemerkt.

Ganz objektiv und ohne Bezugnahme auf die Regeln der Regeneration betrachtet, liegen die Verhältnisse bei den Hydromedusen so: Daß die durch Knospung sich vermehrenden Hydroidkolonien die oben erwähnte, weitgehende Regenerationsfähigkeit besitzen, hängt aufs engste zusammen mit dem Aufbau ihres Gewebes, ihrer ungeschlechtlichen Fortpflanzungsweise und wobl auch mit dem Bedürfnis für eine solche Fahigkeit in Anbetracht der Sessilität und leichten Verwundbarkeit. Andrerseits ist es erklärlich, das die Regenerationskraft der Generation ${ }^{1}$ ) mangelt, die sich in kleinen, mit komplizierten Einrichtur.gen ausgestatteten Individuen zusammendrängt, welche im Verhältnis zu ihrer eigenen Größe enorme Keimmassen reifen lassen. Die regenerierenden Geschlechtsorgane der Cordylophora lacustris sind weder kompliziert, noch sind bei ihnen die Eimassen uberwiegend groß; sie weichen auch von der geschlechtlichen Generation der anderen Hydroiden beträchtlich durch die einlache Entwicklung ab.

Mit den gewonnenen Erfahrungen zeigt sich, daB die Regeln der Regeneration, die unzweifelhaft zu Recht bestehen, vorläufig doch nur allgemeine Regeln bleiben müssen, und dab sie im großen und ganzen wohl ihre Bedeutung haben, andrerseits aber auch wieder beschränkt sind ind ihre Bedeutung, auf Einzelheiten angewendet, versagt.

Ein glücklicher Umstand fügte es, daß bei den Untersuchungen an den Gonophoren ein Faktor zutage trat, der imstande ist, die Regeneration wesentlich zu beeinflussen. Bei einem bisher unbekannten Hydroiden, Pachycordyle fusca, waren nach jedem operativen Eingriffe Störungen zu bemerken, sogenannte Depressionen. Zu fehlen schienen diese Depressionen nur nach Verletzungen des Stammes der Kolonie; bei Operationen an Hydranten waren sie stets bemerkbar und am stärksten traten sie nach Eingriffen an Gonophoren auf. Mit anderen Worten: je differenzierter das verletzte Gewebe ist, um so stärker ist die Depression. Diese äußert sich wiederum am leichtesten am Hydranten, aber auch an jungen, noch nicht differenzierten Gonophoranlagen. Sie

1) Das Verhälthis zwischen Hydroidpolyp und Meduse resp. sessilem Gonophor ist ein Generationswechsel, bei dem abwechselnd eine ungeschlechtliche (Polyp) und eine geschlechtliche (Meduse) Generation zur Geltung kommen. läßt sich durch den Stamm übertragen, bei sehr starken Depressionswirkungen sogar durch das Wurzelgellecht auf die benachbarten Stämme. Altere Gonophore unterliegen dieser Depression nur sehr schwer. Je schwerer aber irgend ein Gewebekomplex den Wirkungen der Depression anheimfällt, um so länger hält auch ihre Wirkung an. Die Hydranten erholen sich stets sehr schnell davon, während die älteren Gonophore ungleich mehr Zeit dazu beanspruchen. Diese infolge der Operationen auftretenden Depressionen beschränken sich nicht nur auf Pachycordyle allein, sondern sie lassen sich mehr oder weniger deutlich auch an verschiedenen anderen Hydroiden bemerken. Es gibt jedoch auch Formen, bei denen ihre Wirkung sicher nicht vorhanden ist. Diese Depressionen nun lassen vermuten, daß das Regenerationsvermögen prinzipiell tatsächlich vorhanden ist, daB es aber durch die Störungen bei der Verstümmelung selbst an seiner Entfaltung gehindert wird. Bei Pachycordyle wenigstens scheinen die Verhältnisse tatsächlich so zu liegen.

\section{Kleine Mitteilungen.}

Über das Zittern der Blätter. Das Zittern des Espenlaubes und der Blätter auch anderer Bäume zu untersuchen war der Zweck einer im Wintersemester 1913 und Sommersemester 1914 im physikalisehen Institut der $\mathrm{K}$. Bay. Universität Erlangen auf Anregung des Instiłutsleiters Geheimrat $E$. Wiedemann ausgeführten Experimentalarbeit. Es wurden sowohl natürliche Blätter wie auch kỉnstliche aus Metall und Glimmer gefertigte Blatt modelle bezuglich ihres Verhaltens in einem Luftstrahl kleinen, aber auch in einem Luftstrahl grofen Querschnittes untersucht. Die Methode war im wesentlichen die, dab ein Bündel konvergenter Lichtstrahlen auf einen winzigen auf der Blattspreite befestigten Spiegel fiel und von ihm auf eine Mattscheibe reflektisrt wurde, die auch durch eine photographische Platte ersetzt werden konnte. Bewegte sich dann das Biatt, so beschrieb der Schnittpunkt der konvergenten Strahlen auf der Scheibenebene eine leuchtende Kurve. Die so bei den natürlichen Blättern erhaltenen Kurven zeichneten sich đurch größtmögliche Einfachheit aus; es waren ellipsenähnliche oder einfach-8-förmige Tiguren; diese Lissajous-Figuren kamen dadurch $z u$ stande, dak, während das Blatt unter Durchbiegung seines Stieles pendelnde Biegungsschwingungen aus. führte, gleichzeitig die Blattspreite, um die Verlängerung des Stieles sich drehend, Torsionsschwingungen machte. Die Anzahl der Torsions- verhielt sich zur Anzahl der Biegungsschwingungen bei den beobachteten natürlichen Blättern der Espe, der kanadischen Pappel, der Pyramidenpappel, des Birnbaumes, des Tulpenbaumes, des Ahorns wie $1: 1$ oder wie $1: 2$. Als dann die Biegungs- und Torsionsschwingungen in besonderen Apparaten ohne Anblasen getrennt untersucht wurden, zeigte sich, dal das Schwingungszahlenverhältnis angenähert dasselbe war, wie es beim Anblasen gefunden worden war. Die bei diesen Untersuchungen gebrauchten Apparate konnten auch evakuiert werden; dabei zeigte sich, daB auch, nachdem der Luftdruck $z$. B. von $730 \mathrm{~mm}$ auf $15 \mathrm{~mm}$ herabgesetzt war, keine merkliche Änderung im Sehwingungszahlenverhältnis festzu- 